\title{
Simulation Analysis of Ferromagnetic Resonance of Low Magnetic Flux Density-Type PT under Single-Phase Earth Fault of 10kV Power Grid
}

\author{
Lin Gong ${ }^{1}$, a , Xiaorui Hu ${ }^{1}$, Haibo Wang ${ }^{2,}$ b, Xiaoyong Zhang ${ }^{1}$, Fei Xiang ${ }^{1}$, Jinyu \\ Wang $^{1}$ and Bin Wang ${ }^{1}$ \\ ${ }^{1}$ Electric Power Research Institute, Chongqing Electric Power Company of State Grid, Chongqing \\ 400044, China \\ ${ }^{2}$ Chongqing University, Chongqing 400044, China

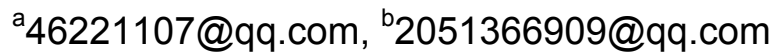

Keywords: Low Magnetic Flux Density-Type Potential Transformer; Ferromagnetic Resonance; Single-Phase Grounding; Potential Transformer Parallel; Simulink.

\begin{abstract}
In a neutral nongrounding power system, the excitation impedance of an electromagnetic potential transformer (PT) and a ground capacitance power transmission line of an electric power system can form a resonant circuit that causes ferroresonance and endangers the security and stability of the power system. This study analyzes a theory of ferromagnetic resonance that occurs under a single-phase earth fault in the power system of a PT, and Simulink is used to establish a simulation model of PT ferromagnetic resonance. Through this model, Simulink conducts an analysis of the ferromagnetic resonance simulation for the isolated operation of an ordinary PT and a low magnetic flux density-type PT, the parallel operation of these two PTs, and the parallel operation of two low magnetic flux density-type PTs. Simulation results show that ferromagnetic resonance phenomenon does not occur easily in a PT with good excitation characteristics. However, ferromagnetic resonance can be generated easily in PT parallel connections.
\end{abstract}

\section{Introduction}

Resonance results in system overvoltage, endangers weak-insulation equipment, causes accidents of flashover and trip, and leads to lightning arrester explosion, fuse failure of potential transformer (PT) high-pressure side fuse, PT overcurrent, and burning loss or explosion accidents ${ }^{[1-4]}$. PT resonance problem has been seriously threatening the safety and stability of power grid operations. It is also one of the causes of some major accidents in a power system. In view of the PT resonance problem in a power distribution network, the ferromagnetic resonance of the system can be alleviated slightly with low magnetic flux density-type PT. However, electrical accidents caused by the resonance of low magnetic flux density-type PTs still occur in practical application.

This study conducts a simulated analysis of the ferromagnetic resonance of an ordinary PT and a low magnetic flux density-type PT to provide certain theoretical guidance for the analysis of PT ferromagnetic resonance in the application. In a power system, a PT is connected on the bus of an electric generator or a substation to monitor the voltage to earth of buses in the power station and substation ${ }^{[1]}$. After primary winding, a star connection is conducted on the PT by directly grounding the neutral point. When the power grid is excited by some perturbations, the excitation impedance of the PT and the ground capacitance of the system form a nonlinear resonance circuit, thereby generating ferromagnetic phenomenon. Ferromagnetic resonance overvoltage caused by electromagnetic PT is the most common internal overvoltage in a neutral nongrounding system and causes the majority of accidents.

\section{Ferromagnetic Resonance Principle of a Three-Phase PT}

In a power system, the PT is usually installed on the bus of a three-phase voltage; thus, ferromagnetic resonance is a three-phase ferromagnetic resonance ${ }^{[5]}$. In a neutral nongrounding 
system, the PT is usually connected in the mode ${ }^{[6,7]}$ of "star-star-triangle." Given the nonlinear characteristics $^{[8]}$ of an PT iron core, the magnetic inductance value changes with the variation of current, wave distortion occurs under the influence of alternating current power supply, and the generated ferromagnetic resonance does not have a fixed resonant frequency ${ }^{[9-11]}$. In the same electric circuit, frequency dividing resonance (harmonic waves such as $1 / 2,1 / 3,1 / 5$, and 2/5 of base frequency), fundamental resonance $(50 \mathrm{~Hz})$, and high-frequency resonance (harmonic waves such as $2^{\text {nd }}, 3^{\text {rd }}$, and $5^{\text {th }}$ orders) may occur ${ }^{[12]}$. Frequency $f$, which experiences resonance, is calculated by using Equation (1), that is, it is decided by the equivalent capacitance $C$ (usually the earth capacitance of a circuit) and the equivalent inductance L (ground inductance of PT) of the oscillating circuit.

$$
f=\frac{1}{2 \pi \sqrt{L C}}
$$

The ground capacitance of each phase is the same as the characteristics of a three-phase PT when the system operates normally, and the PT and neutral point of the power supply have no displacement. The current that flows through the PT changes when a large-disturbance motion appears in an electric power system and the nonlinear iron core of the PT may saturate; thus, a ferromagnetic resonance is formed with the ground capacitance of the circuit, and resonance overvoltage is generated ${ }^{[13,14]}$. Practical operating experience shows that in neutral nongrounding power grids that are not more than $35 \mathrm{kV}$, the PT connected on the bus of an electric generator or substation forms a star connection after primary winding, and the neutral point is grounded. Figures 1 and 2 show the wiring diagram of a three-phase PT and its equivalent circuit diagram.

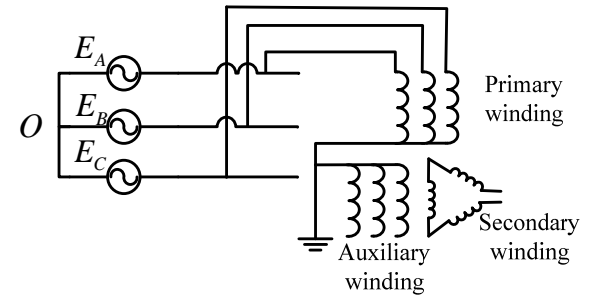

Fig. 1 Wiring diagram of a PT.

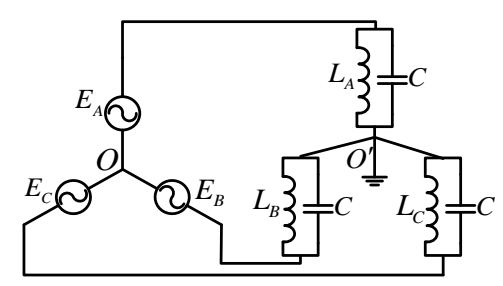

Fig. 2 Equivalent circuit diagram of a PT.

$E_{\mathrm{A}}, E_{\mathrm{B}}$, and $E_{\mathrm{C}}$ in Figures 1 and 2 are the three-phase supply potential and ground capacitance (the set values of the ground capacitance of each phase are the same) of the power transmission line; $L_{\mathrm{A}}$, $L_{\mathrm{B}}$, and $L_{\mathrm{C}}$ are the inductances of a three-phase voltage transformer; $O$ and $O^{\prime}$ are the neutral points of the power grid and PT, respectively.

The sum of the total charge on the ground capacitance of the power transmission line is zero when the system operates normally. The voltage of the nonfault phase rises to the line voltage when a single-phase earth fault occurs, and the ground capacitance is charged with the electric charge quantity that corresponds to line voltage quantity. During ground fault, the electric charge, which is influenced by the line voltage, circulates between the guide line and the earth while taking touchdown point as the access, thereby forming a capacitance current. After the earth fault disappears, the access between the guide line and the earth on ground capacitance of the guide line is cut off. At this moment, the ground voltage of each phase needs to recover to the phase voltage level of the normal operation. The ground capacitance of the former nonfault phase circuit is charged with the electric charge under the line charge. Given that the touchdown point is cut off, free charge can flow into the earth only through the grounding location of the neutral point of PT's primary winding. At this moment, a large quantity of free charge results in iron core saturation during the process of flowing into the earth. The iron core of PT enters the saturation zone, and the magnetic inductance value decreases rapidly. Thus, the high-pressure side magnetic inductance of PT and ground capacitance of the system may meet the conditions of the resonance, thereby resulting in overvoltage and overcurrent of ferromagnetic resonance. 


\section{Establishing the Simulation Model}

The majority of past simulations adopt nonlinear inductance or segmented inductance to simulate PT while neglecting the influence of iron loss and leakage inductance of PT on simulation results. This study adopts a saturation transformer in Simulink to simulate PT by considering the influence of the iron loss and leakage inductance of PT on the simulation results. In view of the $10 \mathrm{kV}$ power grid network, this thesis initially establishes a mathematical model of PT. Then, the ferromagnetic resonance simulation model of PT is established. Fig. 3 illustrates the internal equivalent circuit of PT.

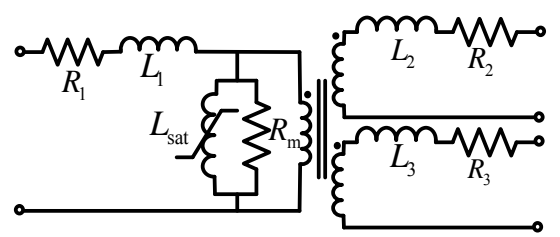

Fig. 3 Internal equivalent circuit of a PT.

In Figure $3, R_{1}, R_{2}$, and $R_{3}$ are the resistances of the primary, secondary, and auxiliary sides of the PT, respectively; $L_{1}, L_{2}$, and $L_{3}$ are the leakage inductances of the primary, secondary, and auxiliary sides of the PT, respectively. $R_{\mathrm{m}}$ is the iron loss of PT, and $L_{\mathrm{sat}}$ is nonlinear inductance with iron core. In the simulation process, this inductance value varies with the set VA characteristic curve. The nonlinear characteristics of PT are simulated well while considering that winding resistance, saturation of leakage inductance, and transformer model can approach the real condition of a PT in an actual operation. The simulation in this study adopts the VA performance data of JDZX16-10RG PT.

The PT model established under Simulink needs the relationship between current per-unit value and magnetic flux per-unit value. Thus, we convert the model's performance data of excitation saturation. The following are the conversion formulas:

Reference value of current: $I_{\text {base }}=\sqrt{2} P_{n} / U_{0}$,

Reference value of magnetic flux: $\Phi_{\text {base }}=\sqrt{2} U_{0} / 2 \pi f_{n}$,

Current per-unit value: $I_{p u}=I / I_{\text {base }}$,

Magnetic flux per-unit value: $\Phi_{p u}=\Phi / \Phi_{\text {base }}=U / U_{0}$,

Reference value of winding: $R_{\text {base }}=U_{n}{ }^{2} / P_{n}$,

Reference value of leakage inductance: $L_{\text {base }}=R_{\text {base }} / 2 \pi f_{n}$,

where $P_{\mathrm{n}}$ is the rated capacity of PT, $20 \mathrm{VA} ; f_{\mathrm{n}}$ is the rated frequency, $50 \mathrm{~Hz} ; U_{0}$ is the rated phase voltage of the system, $5.77 \mathrm{kV}$; and the rated current of PT is $2.8 \mathrm{~mA}$. $I$ and $U$ are the reduction values of the primary side current and the voltage, respectively.

Table 1 presents the valuations of JDZX16-10RG parameters and the leakage inductance of the primary winding resistance. Table 2 presents the measured VA performance data and converted excitation performance data of PT secondary-side reduction.

\begin{tabular}{|c|c|c|}
\hline & Actual Value & Per-Unit Value \\
\hline Resistance & $1727 \Omega$ & 0.00108 \\
\hline Leakage inductance & $68.8 \mathrm{H}$ & 0.013 \\
\hline JDZX16-10RG parameters & $P_{\mathrm{n}}=20 \mathrm{VA} ; P_{\mathrm{n}}=5$ & $I=2.8 \mathrm{~mA} U=5.77$ \\
\hline
\end{tabular}

Table 2 Current and voltage reduction values and P.U. values of current and magnetic flux

\begin{tabular}{cccccccccccccc}
\hline$I / \mathrm{mA}$ & 2.5 & 3.8 & 4.8 & 6.2 & 8.5 & 11.9 & 12.6 & 23.5 & 32.6 & 45.9 & 61.1 & 90.1 & 119 \\
\hline$U / \mathrm{kV}$ & 5.0 & 7.0 & 8.0 & 9.0 & 10 & 10.8 & 11.2 & 11.6 & 11.8 & 12 & 12.2 & 12.7 & 12.6 \\
$I_{\text {pu }}$ & 0.51 & 0.78 & 0.98 & 1.27 & 1.73 & 2.43 & 3.18 & 4.8 & 6.65 & 9.37 & 12.4 & 18.3 & 24.4 \\
$\phi_{\text {pu }}$ & 0.87 & 1.21 & 1.39 & 1.56 & 1.73 & 1.87 & 1.94 & 2.01 & 2.05 & 2.08 & 2.11 & 2.15 & 2.18 \\
\hline
\end{tabular}


On the basis of the preceding data, a ferromagnetic resonance simulation model of the neutral nongrounding system is established in Simulink, as shown in Fig. 4. The ideal power supply is $10 \mathrm{kV}$ AC power supply. LA, LB, and LC are the three-phase line parameters, and different parameters mean different lengths of electric transmission lines connected to the system. The PTs on the three-phase buses are starlike connections featured by connected buses after primary winding and neutral-point solid grounding. A closed switch indicates that a phase single-phase earth fault occurs in the system, whereas an open switch indicates that the earth fault disappears. The neutral-point voltage of the system measured with an oscillorange is the zero-sequence voltage of the system.

The preceding theoretical analysis indicates that the single-phase earth fault, as a condition that stimulates ferromagnetic resonance, alters the length of the electric transmission line (namely, the ground capacitance values of the electric transmission line are altered) by adjusting the capacitance parameter values in the simulation model. If the PT is stimulated under a single-phase earth fault, it may probably enter the saturation zone, and the magnetic inductance value decreases rapidly. Meanwhile, the ground capacitance values of the circuit are altered, and the process of the system generating ferromagnetic resonance is simulated with the appropriate cooperation of the system parameters, thereby finding the cooperating zone of the system parameters when the resonance occurs.

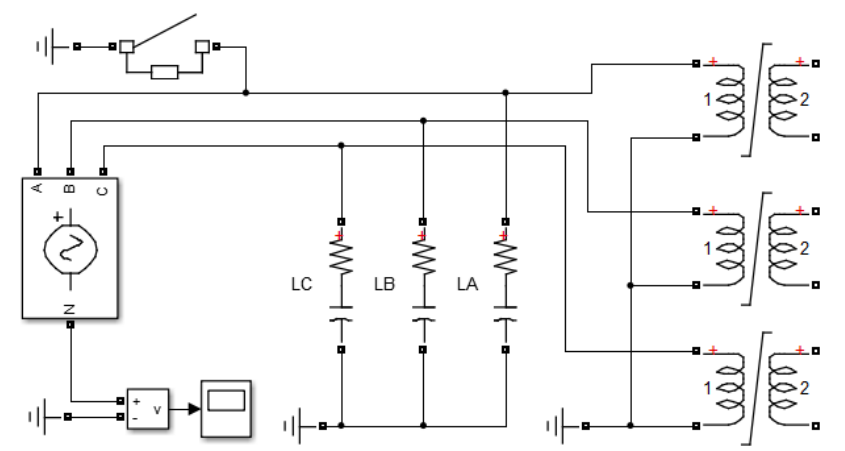

Fig. 4 Simulation model of ferromagnetic resonance.

On the basis of the VA characteristic data of JDZX16-10RG PT, the inductance range that corresponds to the measured VA characteristics can be solved according to Formula (2), in which $L$ is the inductance value, $U$ is the voltage value, $I$ is the current value, and $f_{0}$ is $50 \mathrm{~Hz}$. Under the minimum data pair, the current, voltage, and corresponding inductance values are $2.5 \mathrm{~mA}, 5 \mathrm{kV}$, and $6369 \mathrm{H}$, respectively. Under the maximum data pair, the current, voltage, and corresponding inductance values are $119.7 \mathrm{~mA}, 12.6 \mathrm{kV}$, and $335 \mathrm{H}$, respectively.

$$
L=\frac{U}{2 \pi f_{0} I}
$$

The theoretical ground capacitance value of the circuit is obtained with Formula (2) when the ferromagnetic resonances under different frequencies occur within the inductance range of PT, as shown in Table 3.

Table 3 Theoretical capacitance value under different resonance frequencies

\begin{tabular}{ccc}
\hline Resonant Frequency/Hz & Inductance/H & Capacitance $/ \mathrm{nF}$ \\
\hline \multirow{2}{*}{15} & 335 & 336 \\
& 6369 & 17.7 \\
25 & 335 & 121 \\
& 6369 & 6.36 \\
\multirow{2}{*}{50} & 335 & 30.2 \\
& 6369 & 1.59 \\
\hline
\end{tabular}




\section{Simulation Result and Analysis}

\subsection{Single-PT Operation}

Any excitation condition that causes busbar voltage fluctuation may generate resonance overvoltage and overcurrent. The occurrence of single-phase ground fault and fault recovery is the major reason that stimulates ferromagnetic resonance. In the established simulation model, the capacitance range is regulated when a single-phase ground fault occurs and the ideal power supply phase position is zero upon fault recovery. The stable resonance never occurs but with a slight overshoot current, and the overshoot current oscillation decreases rapidly to zero.

A single-phase ground fault occurs when the system is connected with only one ordinary PT, and the ideal power supply phase position is 80 upon fault recovery. The range of capacitance changes continuously, and simulation results are shown in Figs. 5 and 6. In the simulation, a ground fault occurs when the switch is set at $0.06 \mathrm{~s}$, and it recovers at $0.16 \mathrm{~s}$. Figs. 5(a) and 5(b) illustrate zero-sequence voltage waveform under base-frequency resonance and dividing-frequency resonance, respectively $(15 \mathrm{~Hz})$. Figs. 6(a) and 6(b) illustrate the current waveform under base-frequency resonance and dividing-frequency resonance, respectively $(15 \mathrm{~Hz})$.

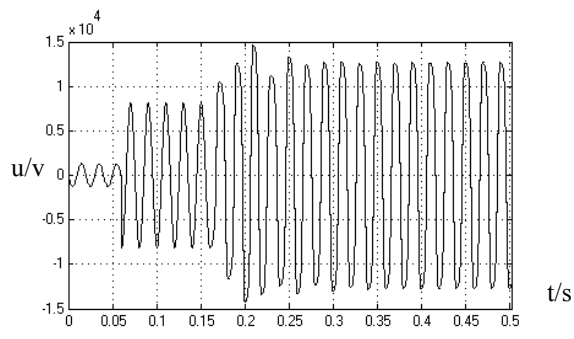

(a)

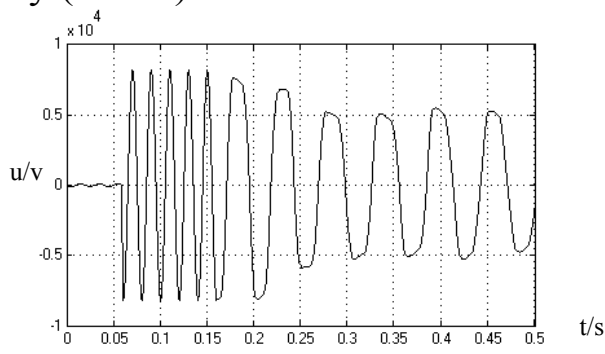

(b)

Fig. 5 Zero-sequence voltage waveform of base-frequency and dividing-frequency resonance.

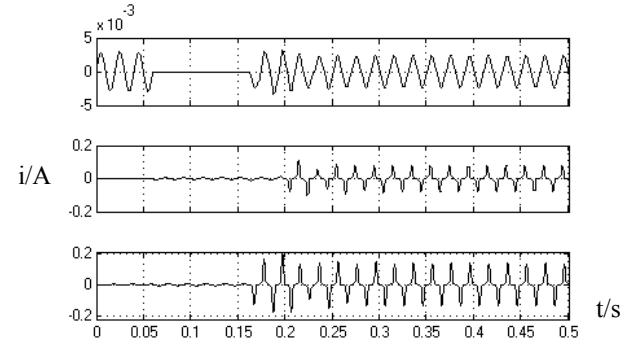

(a)

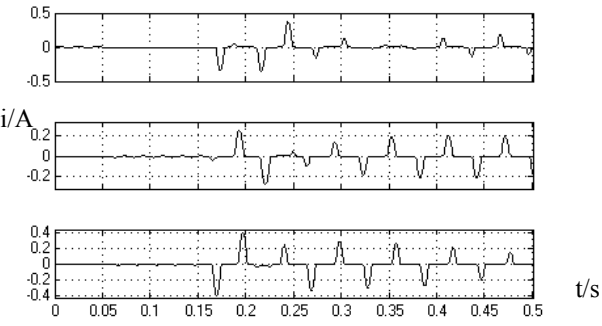

(b)

Fig. 6 PT Current waveform of base-frequency and dividing-frequency resonance.

After the earth fault occurs, zero-sequence voltage is an inverse phase of fault phase voltage. At this moment, the current that flows through the transformer increases but remains in the linear region. An oscillation occurs after the earth fault recovers, and it enters base-frequency resonance rapidly. At this moment, the transformer enters deep-saturation region, and the current increases. Fig. 6(a) shows the maximum value of the overshoot current reaches $135 \mathrm{~mA}$ in a stable base-frequency resonance, which is 48.1 times higher than that of the transformer current under normal operation. The simulation results in Fig. 5(b) indicate that the overshoot current reaches $230 \mathrm{~mA}$ during dividing-frequency resonance, which is 82.14 times higher than the transformer current under normal operation.

High-frequency resonance does not exist in the simulation. However, base-frequency and dividing-frequency resonances exist. The capacitance value range of base-frequency resonance is 3 $\mathrm{nF}$ to $8 \mathrm{nF}$. The capacitance value range of dividing-frequency resonance $(15 \mathrm{~Hz})$ is $99 \mathrm{nF}$ to $101 \mathrm{nF}$, and the simulation result is basically identical to the theoretical calculation range of the capacitance shown in Table 3. The simulation result conforms to the tendency that with increased capacitance, the resonance changes from high-frequency resonance to base-frequency resonance to dividing-frequency resonance. The occurrence of ferromagnetic resonance phenomenon under excitation conditions of PT is verified, and the correctness of the established simulation model is testified. 
The low magnetic flux saturation voltage transformer exhibits good excitation characteristic linearity and high saturation points. Saturating is more difficult for this type of transformer than the ordinary voltage transformer. Thus, its application in the power system can effectively reduce the probability of ferromagnetic resonance phenomenon. However, the potential risk of the resonance of a low magnetic flux saturation voltage transformer remains. This study alters the saturation points of the excitation characteristics on the basis of the excitation characteristics of an ordinary voltage transformer, and the parameter cooperating zone is investigated when ferromagnetic resonance occurs in a low magnetic flux saturation voltage transformer.

The excitation characteristic curve after the per-unit value of the magnetic flow of JDZX16-10RG PT excitation characteristics is elevated to 1.2 times and 1.5 times is shown in Fig. 7. This study conducts a comparative simulation of a low magnetic flux-type PT and an ordinary PT under the same simulation conditions. A comparative analysis of the simulation results is shown in Table 4.

Simulation results show that high-frequency resonance does not occur under the three situations, and base-frequency resonance appears only when the ordinary PT is operated. For comparison, the results in the table are data under the given capacitance value. The following is the PT resonance capacitance range under the original excitation characteristics: base-frequency resonance, $3 \mathrm{nF}$ to 8 $\mathrm{nF}$; dividing-frequency resonance $(15 \mathrm{~Hz}), 99 \mathrm{nF}$ to $101 \mathrm{nF}$. Dividing-frequency resonance $(15 \mathrm{~Hz})$ occurs only when the PT excitation characteristic is improved by 1.2 times. The capacitance value range is $88 \mathrm{nF}$ to $90 \mathrm{nF}$.

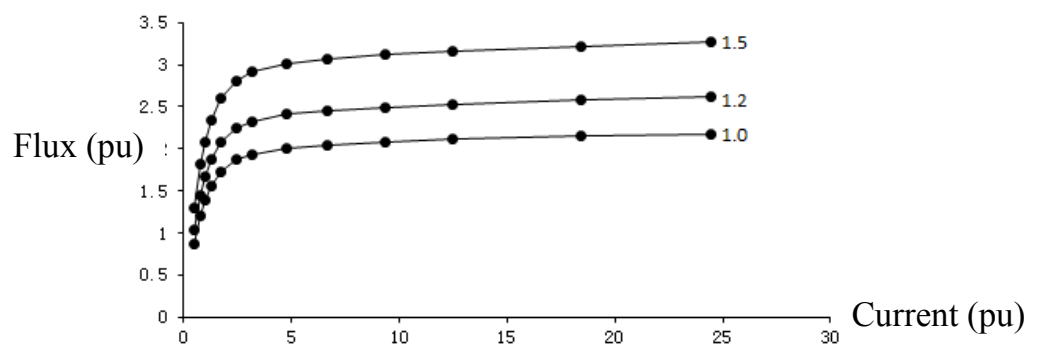

Fig. 7 Improved excitation characteristic curve of PT.

Table 4 Comparison among simulation results under different excitation characteristic PT

\begin{tabular}{ccccccc}
\hline Times & $\begin{array}{c}\text { Resonance } \\
\text { Type }\end{array}$ & $\mathrm{C} / \mathrm{nF}$ & Overcurrent/mA & Overvoltage/kV & $\begin{array}{c}\text { Overcurrent } \\
\text { times }\left(I / I_{0}\right)\end{array}$ & $\begin{array}{c}\text { Overvoltage } \\
\text { times }\left(U / U_{0}\right)\end{array}$ \\
\hline 1 & Base & 8 & 135 & 19.7 & 48.21 & 2.41 \\
1 & Dividing & 100 & 230 & 13.1 & 82.14 & 1.61 \\
1.2 & Dividing & 90 & 255 & 14.5 & 91.07 & 1.78 \\
1.5 & No resonance occurs in the simulation process. Only damped oscillation occurs, and it \\
& \multicolumn{7}{c}{ decreases and disappears rapidly. } \\
\hline
\end{tabular}

Simulation results show that only dividing-frequency resonance occurs in PT when the excitation characteristics are improved by 1.2 times. No resonance but damped oscillation of PT occurs when the excitation characteristics are improved by up to 1.5. The PT with ordinary excitation characteristics and that with 1.2 times better excitation characteristics have a dividing-frequency resonance of $15 \mathrm{~Hz}$. Both overvoltage and overcurrent values of the PT with 1.2 times better excitation characteristics are great. In the theoretical analysis, good excitation characteristic of PT corresponds to infrequent occurrence of ferromagnetic resonance. Once ferromagnetic resonance occurs, the overvoltage and overcurrent increase. The experimental result is identical to the result of theoretical analysis, thereby verifying the correctness of the theoretical analysis and provides the theoretical basis for the actual application of low magnetic flux antisaturation-type PT.

\subsection{PT Parallel Operation}

In general, two PTs are parallel connected on the same bus in a power system. In this study, we conduct a simulation analysis of a situation in which the same bus simultaneously connects low magnetic flux-type PT and ordinary PT, and a situation in which two low magnetic flux-type PTs are parallel connected under the same conditions. The simulation results are shown in Table 5. 
Table 5 Simulation results of parallel-connected PTs of different excitation characteristics

\begin{tabular}{c|c|c|c|c|c|c}
\hline $\begin{array}{c}\text { Parallel } \\
\text { Type }\end{array}$ & $\begin{array}{c}\text { Resonance } \\
\text { Type }\end{array}$ & $\mathrm{C} / \mathrm{nF}$ & $\begin{array}{c}\text { Overcurrent } \\
/ \mathrm{mA}\end{array}$ & $\begin{array}{c}\text { Overvoltage } \\
/ \mathrm{kV}\end{array}$ & $\begin{array}{c}\text { Overcurrent } \\
\text { times }\left(I / I_{0}\right)\end{array}$ & $\begin{array}{c}\text { Overvoltage } \\
\text { times }\left(U / U_{0}\right)\end{array}$ \\
\hline $1+1.2$ & Base & 10 & $132 / 31.8$ & 19.5 & $47.14 / 11.36$ & 2.39 \\
\hline 1 & Dividing & 122 & $405 / 282$ & 19.2 & $144.62 / 100$. & 2.35 \\
\hline $1.2+1.5$ & Dividing & 135 & $263 / 138$ & 14.8 & $93.93 / 49.28$ & 1.81
\end{tabular}

Notes: " $1+1.2$ " indicates that the PT with ordinary excitation characteristic and the PT with an improved excitation characteristic by 1.2 times are parallel connected the same bus. " $1.2+1.5$ " indicates that the PT with an improved excitation characteristic by 1.2 times and the PT with an improved excitation characteristic by 1.5 times are parallel connected on the same bus. "132/31.8" represents the over currents that flow through the PT with ordinary excitation characteristic and through the PT with an improved excitation characteristic by 1.2 times, respectively.

The simulation results show that a high-frequency resonance does not occur in the PT parallel connection simulation, and base-frequency resonance exists only in the parallel connections of the ordinary PT and low magnetic flux-type PT. For comparison, the results in the table are the data under the given capacitance value. The following are the capacitance value ranges of the parallel connection of " $1+1.2$ "-type cooperating PTs: base-frequency resonance, $3 \mathrm{nF}$ to $11 \mathrm{nF}$; and dividing-frequency resonance $(25 \mathrm{~Hz}), 120 \mathrm{nF}$ to $123 \mathrm{nF}$. Only dividing-frequency resonance $(15 \mathrm{~Hz})$ occurs in the parallel connection of " $1.2+1.5$ "-type cooperating PTs, and its capacitance range is $132 \mathrm{nF}$ to 136 $\mathrm{nF}$.

Figs. 8(a) and 8(b) illustrate the simulation results when the parallel-connected PTs are operated. Fig. 8(a) illustrates the current waveform that flows through the PT with 1.2 times better excitation characteristics. Fig. 9(b) illustrates the current waveform that flows through the PT with 1.5 times better excitation characteristics. As shown in Table 5, 263mA and $138 \mathrm{~mA}$ are the maximum impulse currents that flow through the two PTs under stable resonance. Figs. 8(a) and 8(b) show that the current that flows through the PT with 1.2 times better excitation characteristics at the same time the resonance occurs is higher than that which flows through the PT with 1.5 times better excitation characteristics. This observation indicates a low current that flows through the PT with better excitation characteristics when ferromagnetic resonance occurs under the parallel connection of PTs.

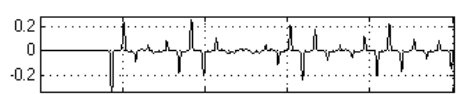

i/A
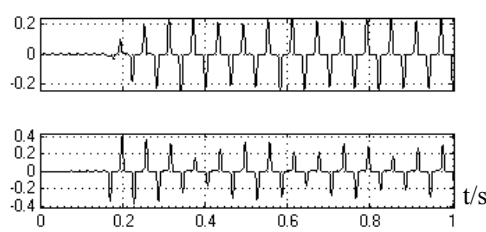

(a) 1.2 times
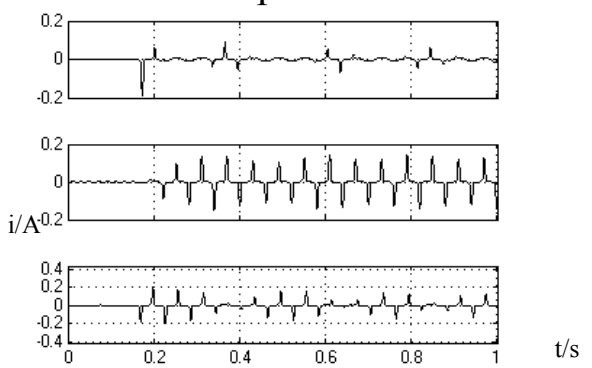

(b) 1.5 times

Fig. 8 Current waveform with 1.2 and 1.5 times excitation characteristics.

A comparison between the simulation results in Tables 4 and 5 indicates that ferromagnetic resonance does not occur when the PT with 1.5 times better excitation characteristics is operated separately, and dividing-frequency resonance occurs when "1 +1.2 "-type cooperating PTs are in parallel operation. Under the same simulation conditions, the PT that has no resonance phenomenon when it operates alone exhibits ferromagnetic resonance under parallel operation. This finding indicates that PT parallel operation can generate ferromagnetic resonance easily.

\section{Summary}

On the basis of the simulation result analysis of the PT ferromagnetic resonance model established in this study, the following conclusions are drawn:

1. The excitation characteristic of a PT plays a significant role in the occurrence of ferromagnetic resonance. The antisaturation performance of a low magnetic flux-type PT is better than that of an ordinary PT. Ferromagnetic resonance does not occur easily in a low magnetic flux-type PT. 
Ferromagnetic resonance does not occur in PT with good excitation characteristics under general excitation conditions.

2. In the simulation, the ordinary PT and the PT with 1.2 times excitation characteristic have the same frequency resonances. The overvoltage and overcurrent of low magnetic flux-type PT are low. This finding indicates that when the ferromagnetic resonances of the same frequency occur, the overvoltage and overcurrent of the PT with good excitation characteristics are low.

3. Under the same excitation conditions, the PT that does not experience ferromagnetic resonance when it operates alone may experience ferromagnetic resonance under a parallel operation. This finding indicates that ferromagnetic resonance can be generated easily in PT parallel connection.

\section{References}

[1]. Mou Daohuai, Li Yusheng, Ma Yuliang, et al. Electrical Part of Power Plant [M]. Chongqing: Chongqing University Publisher, 2006

[2]. Tang xingzuo, High Voltage Technology [M]. Chongqing: Chongqing University Publisher, 1996.

[3]. Zhou Lixia, Yin Zhongdong, Zhang Li. Research on Principle of PT Resonance in Distribution Power System and Its Suppression [J]. Transactions of China Electrotechnical, 2007, 22(5): 153-158.

[4]. Sima Wenxia, Guo Fei, Yang Qing, et al. Simulative analysis of its suppression ferroresonance. Electric Power Automation Equipment, 2007, 27(6): 22-26.

[5]. Chen Yan, Power System Steady State Analysis [M], $3^{\text {th }}$ edition, Beijing: China Electric Power Press, 2007

[6]. Lin Li, Wang Junbing, Tang Fengying, et al. Simulation and computational analysis on potential transformer damage in $10 \mathrm{kV}$ system[J]. Power System Protection and Control, 2012, 40(17): 51-55.

[7]. Lin Li, He Yue, Wang Junbing, et al. Mechanism of Potential Transformer Damaged in Ungrounded Neutral Power System[J]. High Voltage Engineering, 2013, 39(5): 1114-1120.

[8]. Tang Yunmiu, Electrical Motor [M], $4^{\text {th }}$ edition, Beijing: China Machine Press, 2011

[9]. Weng Limim, Chen Lingxin, Jin Jianfeng. Characteristic and Suppression of Ferrcrresonance of Electromagnetic PT in Distribution Network[J]. Relay, 2004, 32(20): 40-42.

[10]. Yang Binwen, Li Wensheng. Cause of PT Ferroresonanceand Countermeasures [J]. Electric Power automation quipment, 2010, 30(3): 134-136, 148.

[11]. Mei Chenglin, Zhang Chaoshu. Analysis of voltage transformer ferroresonance[J]. Power system technology, 2008, 32(2): 311-313.

[12]. Peterson HA. Transients in Power Systerms[M]. New York: John Wiley\&Sons, Inc, 1951.

[13]. L.X.Zhou and Z.D.Yin, Member, IEEE. Research on PT Ferromagnetic Resonance Region and Controllable Damping[J]. Power Tech, 2007, 413-418.

[14]. X.K.Zhu, Y.H. Yang et al. Study on Ferro-resonance due to Electromagnetic PT in Ungrounded Neutral System[C]. 2004 International Conference on Power System Technology, 2004. 